\title{
Control of Quantum States by Decoherence
}

\author{
L. Accardi and K. Imafuku
}

November 27, 2009

\begin{abstract}
We propose a new technique to control quantum states by exploiting the decoherence due to the coupling with environment. With this technique we can get any target state, in a stable way and in an exponentially small time, as a stationary state of a semigroup (master equation) canonically derived from a microscopic Hamiltonian model. The stationary state of the system depends (i) on the interaction with the environment; (ii) on the initial state of the environment and it "inherits" some properties of it in a sense that will be explained in sec. 5 of the present paper. Moreover this is true not only for by thermal or vacuum environments but also for more general non-equilibrium environments. We prove that, by appropriately choosing this control parametes (interaction system-environment, initial environment state) one can drive the system to an arbitrary preassigned quantum state.
\end{abstract}

\section{Introduction}

Recent developments of technology greatly improved our ability to control individual quantum systems. This brings quantum technology beyond academic research to the level of concrete industrial programs $[1,2]$. For the requirements of any quantum technology the requirement of stability is essential: it is not only required that at time $T$ the system is in a given quantum state, but also that it remains in this state sufficiently long time to allow the manipulations required by quantum computation. One possible way to achieve this goal is to exploit a general principle of the stochastic limit [3, 4, 5, 6], namely: under explicit and easily realizable conditions, the interaction of a quantum field with a discrete system (e.g. an $N$-level atom) drives the system to a stationary state which is uniquely determined by the initial state of the field and by the form of the interaction.

Already now many manipulations on microscopic objects are achieved through their interaction with appropriate fields (for example, a creation of entangled state with ion trap (ions + phonon) or with cavity QED (atom + EM field) have been already reported $[7,8,9,10]$.) The scenario we are proposing generalizes this approach by extending it to a large class of interactions and integrates it with the additional requirement of stability. In some sense, this scenario realizes the converse program of the stochastic limit: there one starts from a given interaction and a given state of the field and looks for the corresponding Langevin and master equations and their stationary states. Here one starts from a state of the system, say atom or lattice, and looks for an interaction and an initial 
state of the field such that the associated master equation will drive the system to the given state. In other words: in our approach the initial state of the field and the interaction Hamiltonian are seen as control parameters.

The advantage of the stochastic limit approach is that it gives a quite explicit description of the parameters which control the final state of the system. Therefore, if we are able to act on these parameters by suitably choosing the initial state of the field and the interaction, we could drive the system, in a stable way and in an exponentially small time, to a large class (in principle, any) of pre-assigned states.

In this paper, we consider the conditions on the microscopic systems which realize the above scenario. As shown in sec.2, it is easy to write down a general master equation which drives any quantum state to a given target state. Our interest in this paper is to derive this master equation from a microscopic Hamiltonian model. This allows to make clear the physical conditions to realize this control. From sec. 3 we show that as far as the system degrees of freedom are finite for any target states we can always find an appropriate microscopic model. We also discuss the concrete conditions on the initial state of the environment. For a given target state, the initial state of the environment can be chosen in a variety of ways whereas the condition for the interaction are more restrictive. This means that, in real experimental situations, one can choose an easily realizable initial state which depends on the individual case. In sec. 5, we show that the system has the "assimilation property", i.e. if the initial state of the environment is non-equilibrium (resp. equilibrium or vacuum) for the free environment Hamiltonian, then the system is driven to a non-equilibrium (resp. equilibrium or ground) state for the free system Hamiltonian. In the equilibrium case, this assimilation property is true for rather general models, but in non-equilibrium case, it appears as a consequence of the specific choice of the interaction. In sec. 6 we discuss purification as a special case of our general framework: any pure state can be obtained by a general assimilation property with a vacuum environment. As an example in sec. 7 , the singlet state of two spin- $1 / 2$ particles.

\section{A master equation driving to a pre-assigned state}

Let us consider a master equation

$$
\frac{d}{d t} \rho(t)=\mathcal{L}_{*} \rho(t)
$$

with GKSL generator $[12,13]$

$$
\mathcal{L}_{*} \rho(t)=-i[H, \rho(t)]-\gamma \sum_{j \neq k} p_{j}\left(\frac{1}{2}\left\{L_{j k}^{\dagger} L_{j k}, \rho(t)\right\}-L_{j k} \rho_{t} L_{j k}^{\dagger}\right)
$$

with $H, p_{j}, L_{j k}$ given respectively by

$$
H=\sum_{j} \epsilon_{j}\left|\mu_{j}\right\rangle\left\langle\mu_{j}\right|, \quad \sum_{j} p_{j}=1, \quad\left(p_{j} \geq 0\right),
$$


Notice that

$$
L_{j k}=\left|\mu_{j}\right\rangle\left\langle\mu_{k}\left|, \quad L_{j k}^{\dagger}=\right| \mu_{k}\right\rangle\left\langle\mu_{j}\right|
$$

$$
\sum_{j k} p_{j} L_{j k}^{\dagger} L_{j k}=1, \quad \sum_{j k} p_{j} L_{j k} \rho L_{j k}^{\dagger}=\sum_{j} p_{j}\left|\mu_{j}\right\rangle\left\langle\mu_{j}\right| .
$$

Therefore one can rewrite the master equation as

$$
\begin{aligned}
\mathcal{L}_{*} \rho(t)= & -i[H, \rho(t)]-\gamma \sum_{j, k} p_{j}\left(\frac{1}{2}\left\{L_{j k}^{\dagger} L_{j k}, \rho(t)\right\}-L_{j k} \rho_{t} L_{j k}^{\dagger}\right) \\
& +\gamma \sum_{j} p_{j}\left(\frac{1}{2}\left\{L_{j j}^{\dagger} L_{j j}, \rho(t)\right\}-L_{j j} \rho_{t} L_{j j}^{\dagger}\right) \\
= & -i[H, \rho(t)]-\gamma(\rho(t)-\mu) \\
& +\gamma \sum_{j} p_{j}\left(\frac{1}{2}\left\{\left|\mu_{j}\right\rangle\left\langle\mu_{j}\right|, \rho(t)\right\}-\left\langle\mu_{j}|\rho(t)| \mu_{j}\right\rangle\left|\mu_{j}\right\rangle\left\langle\mu_{j}\right|\right),
\end{aligned}
$$

where

$$
\mu:=\sum_{j} p_{j}\left|\mu_{j}\right\rangle\left\langle\mu_{j}\right|
$$

One can see that, denoting $\rho_{m n}(t)=\left\langle\mu_{m}|\rho(t)| \mu_{n}\right\rangle$, (2) becomes equivalent to

$$
\begin{gathered}
\frac{d}{d t} \rho_{m m}(t)=\gamma\left(p_{m}-\rho_{m m}(t)\right) \\
\frac{d}{d t} \rho_{m n}(t)=\left(i\left(\epsilon_{n}-\epsilon_{m}\right)-\gamma\left(1-\frac{p_{m}+p_{n}}{2}\right)\right) \rho_{m n}(t) .
\end{gathered}
$$

Thus the state of the system is driven to the final state (7), i.e.

$$
\rho(t) \rightarrow \mu, \quad \text { as } t \rightarrow \infty
$$

Notice that the convergence in (10) is exponential and its speed of convergence is given by $\gamma^{-1}$ which is thus interpreted as life time of the initial state $\rho(0)$. It means that if can we realize a physical system which is described by this master equation, we can control a system so that its state is driven to the target state $\mu$. In the following section, we prove that the stochastic limit technique [ ] allows to solve this problem, i.e. to construct microscopic models from which to derive the master equation (8), (9) and the explicit form of the life time $\gamma$.

Moreover these microscopic models are based on relatively simple, dipole type, interaction Hamiltonians. More precisely we prove that, given any state $\mu$ (cf. (7)) of a finite dimensional system, one can find an appropriate dipole type coupling of this system with a quantum field (concretely this means to specify the form of the operator $D$ in the interaction (13)) such that the system will be asymptotically driven to the given state (in the sense of (10)) whatever its initial state is.

The explicit from of the operator $D$, as a function of the target state, will be given in section (5) below.

The practical implementation of the corresponding dipole interaction will of course depend on the system and on the target state and has to be discussed case by case. However just the possibility, in principle, of such a "control by decoherence" is quite a non trivial fact. We prove this possibility and we give an explicit and constructive description of the solutions. 


\section{A Microscopic Model}

In the following sections, we show how to derive the master equation of sec.2 from a microscopic Hamiltonian model through the stochastic limit $[3,4,5]$.

We consider the following Hamiltonian system:

$$
H_{t o t}=H_{S}+H_{B}+\lambda H_{I}, \quad H_{0}=H_{S}+H_{B}
$$

where, for the same $\mu_{j}$ as in (3), (7)

$$
\begin{gathered}
H_{S}=\sum_{j} E_{j}\left|\mu_{j}\right\rangle\left\langle\mu_{j}\right|, \quad H_{B}=\int d k \omega(k) a_{k}^{\dagger} a_{k}, \quad\left[a_{k}, a_{k^{\prime}}^{\dagger}\right]=\delta\left(k-k^{\prime}\right) \\
H_{I}=\int d k\left(g(k) D a_{k}^{\dagger}+g^{*}(k) D^{\dagger} a_{k}\right)
\end{gathered}
$$

and $D$ is a system operator that will be specified in Section (5). For the initial state of the boson-field, we consider a general mean-zero gaussian state which is represented by the covariance matrix [3]

$$
\left(\begin{array}{cc}
\left\langle a_{k}^{\dagger} a_{k^{\prime}}\right\rangle & \left\langle a_{k} a_{k^{\prime}}\right\rangle \\
\left\langle a_{k}^{\dagger} a_{k^{\prime}}^{\dagger}\right\rangle & \left\langle a_{k} a_{k^{\prime}}^{\dagger}\right\rangle
\end{array}\right)=\left(\begin{array}{cc}
\alpha_{k}^{(+)} \delta\left(k-k^{\prime}\right) & f_{k} \delta\left(k+k^{\prime}\right) \\
f_{k}^{*} \delta\left(k+k^{\prime}\right) & \alpha_{k}^{(-)} \delta\left(k-k^{\prime}\right)
\end{array}\right)
$$

where

$$
\begin{aligned}
\alpha_{k}^{(+)} & =\left|c_{k}\right|^{2} n(k)+\left|s_{k}\right|^{2}(n(k)+1) \\
\alpha_{k}^{(-)} & =\left|c_{k}\right|^{2}(n(k)+1)+\left|s_{k}\right|^{2} n(k) \\
f_{k} & =c_{k} s_{-k}^{*} n(k)+c_{-k} s_{k}^{*}(n(k)+1) .
\end{aligned}
$$

Notice that this is a squeezed state (i.e. $f_{k} \neq 0$ ) which can be obtained from the gauge invariant state of the $\tilde{a}_{k} \tilde{a}_{k}^{\dagger}$-field, with covariance

$$
\left(\begin{array}{cc}
\left\langle\tilde{a}_{k}^{\dagger} \tilde{a}_{k^{\prime}}\right\rangle & \left\langle\tilde{a}_{k} \tilde{a}_{k^{\prime}}\right\rangle \\
\left\langle\tilde{a}_{k}^{\dagger} \tilde{a}_{k^{\prime}}^{\dagger}\right\rangle & \left\langle\tilde{a}_{k} \tilde{a}_{k^{\prime}}^{\dagger}\right\rangle
\end{array}\right)=\left(\begin{array}{cc}
n(k) & 0 \\
0 & n(k)+1
\end{array}\right) \delta\left(k-k^{\prime}\right)
$$

by means of the Bogolyubov transformation

$$
\left(\begin{array}{c}
a_{k} \\
a_{-k}^{\dagger}
\end{array}\right)=\left(\begin{array}{cc}
c_{k}^{*} & s_{k} \\
s_{-k}^{*} & c_{-k}
\end{array}\right)\left(\begin{array}{c}
\tilde{a}_{k} \\
\tilde{a}_{-k}^{\dagger}
\end{array}\right)
$$

(cf. chap. 2 of [3] for details) where

$$
\left|c_{k}\right|^{2}-\left|s_{k}\right|^{2}=1
$$

\section{The Master equation}

In the following discussion, we assume that we can build up the system Hamiltonian $H_{S}$ such that the genericity condition [5]

$$
\omega_{j i}=E_{j}-E_{i}=\omega_{j^{\prime} i^{\prime}} \Leftrightarrow i=i^{\prime}, j=j^{\prime} .
$$


is satisfied. Under this condition, using the stochastic golden rule of [7] (Chap. 5 ), we can obtain the master equation for the reduced density matrix:

$$
\frac{d}{d t} \rho_{t}=+i\left[\Delta, \rho_{t}\right]-\sum_{i \neq j} \Gamma_{i j}\left(\frac{1}{2}\left\{L_{i j}^{\dagger} L_{i j}, \rho_{t}\right\}-L_{i j} \rho_{t} L_{i j}^{\dagger}\right)
$$

where the $L_{i j}$ are given by (29) and

$$
\begin{gathered}
\Delta=\sum_{i j} \Delta_{i j}\left|\mu_{j}\right\rangle\left\langle\mu_{j}\right| \\
\Delta_{i j}=\Delta_{c, i j}^{(-)}+\Delta_{s, i j}^{(+)}-\Delta_{c, j i}^{(+)}-\Delta_{s, j i}^{(-)}, \\
\Gamma_{i j}= \begin{cases}\Gamma_{c, i j}^{(-)}+\Gamma_{s, i j}^{(+)} & \left(E_{j}>E_{i}\right) \\
\Gamma_{c, j i}^{(+)}+\Gamma_{s, j i}^{(-)} & \left(E_{i}>E_{j}\right) .\end{cases} \\
\Gamma_{c, i j}^{(-)}=2 \pi \int d k\left|g_{i j}(k) c_{k}\right|^{2}(n(k)+1) \delta\left(\omega(k)-\omega_{j i}\right) \geq 0 \\
\Gamma_{s, i j}^{(-)}=2 \pi \int d k\left|g_{i j}(k) s_{k}\right|^{2}(n(-k)+1) \delta\left(\omega(k)-\omega_{j i}\right) \geq 0 \\
\Gamma_{c, i j}^{(+)}=2 \pi \int d k\left|g_{i j}(k) c_{k}\right|^{2} n(k) \delta\left(\omega(k)-\omega_{j i}\right) \geq 0 \\
\Gamma_{s, i j}^{(+)}=2 \pi \int d k\left|g_{i j}(k) s_{k}\right|^{2} n(-k) \delta\left(\omega(k)-\omega_{j i}\right) \geq 0
\end{gathered}
$$

Notice that, because of the $\delta$-function in (26)-(29), only those pairs $i j$ with $\omega_{j i}=E_{j}-E_{i}>0$ give a non-trivial contribution since $\omega(k)>0$.

$$
\begin{aligned}
& \Delta_{c, i j}^{(-)}=\text {P.P. } \int d k \frac{\left|g_{i j} c_{k}\right|^{2}(n(k)+1)}{\omega(k)-\omega_{j i}} \\
& \Delta_{s, i j}^{(-)}=\text {P.P. } \int d k \frac{\left|g_{i j} s_{k}\right|^{2}(n(-k)+1)}{\omega(k)-\omega_{j i}} \\
& \Delta_{c, i j}^{(+)}=\text {P.P. } \int d k \frac{\left|g_{i j} c_{k}\right|^{2} n(k)}{\omega(k)-\omega_{j i}} \\
& \Delta_{s, i j}^{(+)}=\text {P.P. } \int d k \frac{\left|g_{i j} s_{k}\right|^{2} n(k)}{\omega(k)-\omega_{j i}}
\end{aligned}
$$

Notice that the $\Gamma_{i j}$ are strictly positive. Moreover if the condition

$$
\Gamma_{i j}=\Gamma_{i}
$$

holds, we can rewrite the master equation (22) as

$$
\frac{d}{d t} \rho_{t}=+i\left[\Delta, \rho_{t}\right]-\Gamma \sum_{i \neq j} \tilde{p}_{i}\left(\frac{1}{2}\left\{L_{i j}^{\dagger} L_{i j}, \rho_{t}\right\}-L_{i j} \rho_{t} L_{i j}^{\dagger}\right)
$$

where

$$
\tilde{p}_{i}=\frac{\Gamma_{i}}{\Gamma}, \quad \Gamma=\sum_{i} \Gamma_{i}
$$


Comparing this master equation with (2), one can verify that the two equations coincide the Hamiltonian part in (36) commutes with the dissipative part whenever $H=-\Delta, \gamma=\Gamma, p_{j}=\tilde{p}_{j}$. Therefore to guarantee the convergence

$$
\rho_{t} \rightarrow \sum_{i} p_{i}\left|\mu_{i}\right\rangle\left\langle\mu_{i}\right|
$$

it is sufficient that condition (35) and the identities

$$
\gamma=\Gamma ; \quad p_{j}=\tilde{p}_{j}
$$

Now, let us consider how to satisfy the condition (35) for a given family $\left\{\tilde{p}_{j}\right\}$. Introducing the notation

$$
\mathcal{N}(k)=\left|s_{k}\right|^{2}(n(-k)+1)+\left|c_{k}\right|^{2} n(k) \geq 0
$$

we can write

$$
\Gamma_{i j}= \begin{cases}2 \pi\left|d_{i j}\right|^{2} \int d k|g(k)|^{2}(\mathcal{N}(k)+1) \delta\left(\omega(k)-\omega_{j i}\right) & \left(E_{j}>E_{i}\right) \\ 2 \pi\left|d_{j i}\right|^{2} \int d k|g(k)|^{2} \mathcal{N}(k) \delta\left(\omega(k)-\omega_{i j}\right) & \left(E_{i}>E_{j}\right)\end{cases}
$$

where

$$
d_{i j}=\left\langle\mu_{i}|D| \mu_{j}\right\rangle .
$$

We will only consider control environment states which satisfy the additional condition which is implied by the set of conditions:

$$
\begin{gathered}
n(k)=n_{0}(\omega(k)) ; \quad c_{k}=c_{0}(\omega(k)) ; \quad s_{k}=s_{0}((\omega(k)) ; \quad \omega(k)=\omega(-k) \\
\mathcal{N}(k)=N(\omega(k))
\end{gathered}
$$

under this condition (41) becomes

$$
\Gamma_{i j}= \begin{cases}\left|d_{i j}\right|^{2} Q_{i j} N_{i j} & \left(E_{j}>E_{i}\right) \\ \left|d_{j i}\right|^{2} Q_{i j}\left(N_{i j}+1\right) & \left(E_{i}>E_{j}\right)\end{cases}
$$

where

$$
Q_{i j}=2 \pi \int d k|g(k)|^{2} \delta\left(\omega(k)-\left|\omega_{j i}\right|\right)>0
$$

and

$$
N_{i j}=N\left(\left|\omega_{j i}\right|\right) .
$$

Our goal is to solve equations (45) in the unknowns $d_{i j}$ (control interaction) and $N_{i j}$ (control environment state) subject to the additional conditions $(35,(37)$ and (39) which introduce the dependence on the target state. The $Q_{i j}$, given by (46), are additional control parameters, but in our case we will consider them as given. Introducing these conditions, (45) becomes equivalent to

$$
\begin{aligned}
& \gamma p_{i}=\left|d_{i j}\right|^{2} Q_{i j} N_{i j} ; \quad \text { for } E_{j}>E_{i} \\
& \gamma p_{i}=\left|d_{j i}\right|^{2} Q_{i j}\left(N_{i j}+1\right) ; \text { for } E_{j}<E_{i}
\end{aligned}
$$

It is clear that, for any choice of the decay rate $\gamma$ and of the target state (i.e. the $p_{i}$ 's) there exist a multiplicity of solutions depending on our choice of the $d_{i j}$, 
$Q_{i j}, N_{i j}$. This variety of choice will be very useful in the explicit construction of the control mechanism.

It should be emphasized that the freedom in the choice of the environment state is ample but not unlimited. For example if the target state is faithful (all $p_{i}>0$ ) then equation (48) shows that the control state cannot be the Fock state (for which $N_{i j}=0$ for all $i, j$ ). In the following sections we will show how to exploit this freedom in some concrete cases.

Let us summarize the properties which the Hamiltonian should have.

1. $H_{S}$ is discrete and is diagonal in the same basis which diagonalizes the target state $\mu$.

2. The spectrum of $H_{S}$ is generic in the sense of (21).

3. $H_{S}$ must have a lowest eigenvalue and the eigenvalues $E_{i}$ of $H_{S}$ must be ordered from the lowest one while the $\tilde{p}_{i}$ are ordered from the largest one.

4. The $d_{i j}=\left\langle\mu_{i}|D| \mu_{j}\right\rangle$ satisfy the equations (48), (49).

\section{Assimilation of the state}

In this section we prove the "assimilation lemma" (1) which shows that, for non degenerate target states, there is a universal relation between the control state of the environment and the target state of the field.

In the final part of this section we explain in what this relation is a natural extension of the fact that an equilibrium environment (at a given temperature for the free evolution) drives a system to an equilibrium state at the same temperature (for its own free evolution)

Lemma 1 Suppose that:

(i) the coefficients $\Gamma_{i j}$ of the master equation (22) satisfy condition (35), so that the master equation (22) takes the form (36), (37).

(ii) The probabilities $\left(\tilde{p}_{i}\right)$ satisfy the conditions

$$
0<\tilde{p}_{i} \neq \tilde{p}_{j} \quad ; \quad \forall i \neq j
$$

Then the $\left(\tilde{p}_{j}\right)$ are decreasing functions of the energies $\left(E_{j}\right)$ and, if condition (43) is satisfied (i.e. the density of quanta in the environment state is a function of the energy density) then the following universal relation holds:

$$
N_{i j}=N\left(E_{i}-E_{j}\right)=\frac{1}{\frac{\tilde{p}_{j}}{\tilde{p}_{i}}-1}=\frac{1}{\frac{\Gamma_{j}}{\Gamma_{i}}-1} ; \quad \forall E_{j}<E_{i}
$$

Remark. The relation (51) is universal in the sense that it is independent of everything but the two energy level involved $\left(E_{i}, E_{j}\right)$ and the pair correlations of the fied. The explicit formula for the quotient $\Gamma_{j} / \Gamma_{i}$,

$$
\frac{\Gamma_{j}}{\Gamma_{i}}=\frac{1+\left|\tanh \left(\omega_{i j}\right)\right|^{2} \frac{n\left(\omega_{i j}\right)}{n\left(\omega_{i j}\right)+1}}{\mid \tanh \left(\left.\omega_{i j}\right|^{2}+\frac{n\left(\omega_{i j}\right)}{n\left(\omega_{i j}\right)+1}\right.}
$$


Proof. Solving (45) for $N_{i j}$ with we

$$
E_{i}>E_{j}
$$

we get

$$
N_{i j}=\frac{\Gamma_{i j}}{Q_{i j}\left|d_{i j}\right|^{2}}, \quad N_{i j}=\frac{\Gamma_{j i}}{Q_{i j}\left|d_{i j}\right|^{2}}-1 .
$$

Therefore (54) is equivalent to

$$
N_{i j}=\frac{\Gamma_{i j}}{Q_{i j}\left|d_{i j}\right|^{2}}=\frac{\Gamma_{j i}}{Q_{i j}\left|d_{i j}\right|^{2}}-1
$$

and, because of $(35),(37)$ this is equivalent to

$$
N_{i j}=\frac{\Gamma \tilde{p}_{i}}{Q_{i j}\left|d_{i j}\right|^{2}}=\frac{\Gamma \tilde{p}_{j}}{Q_{i j}\left|d_{i j}\right|^{2}}-1
$$

From this equation we obtain

$$
\left|d_{i j}\right|^{2}=\frac{\Gamma}{Q_{i j}}\left(\tilde{p}_{j}-\tilde{p}_{i}\right)
$$

Since $\Gamma, Q_{i j} \geq 0$, this and assumption (53) imply that $\tilde{p}_{j}$ is a decreasing function of $E_{j}$. Finally, using (50) and (55) we find

$$
N_{i j}=\frac{\tilde{p}_{i}}{\tilde{p}_{j}-\tilde{p}_{i}}
$$

which is (51). To prove universality notice that the expression of the quotient $\Gamma_{j} / \Gamma_{i}$ in (51) is given by (35), (25), (26)-(29). Therefore assumption (43) implies that, for $E_{i}>E_{j}$

$$
\begin{gathered}
\Gamma_{i}=\Gamma_{i j}=\Gamma_{c, j i}^{(+)}+\Gamma_{s, j i}^{(-)}=\left.2 \pi\left|c_{0}\left(\left|\omega_{j i}\right|\right)^{2} n\left(\left|\omega_{j i}\right|\right)\right| d_{i j}\right|^{2} Q_{i j}+ \\
\left.2 \pi\left|s_{0}\left(\left|\omega_{j i}\right|\right)^{2}\left(n\left(\left|\omega_{j i}\right|\right)+1\right)\right| d_{i j}\right|^{2} Q_{i j} \\
\Gamma_{j}=\Gamma_{j i}=\Gamma_{c, i j}^{(-)}+\Gamma_{s, i j}^{(+)}=2 \pi\left|c_{0}\left(\left|\omega_{j i}\right|\right)\right|^{2}\left(n\left(\left|\omega_{j i}\right|\right)+1\right)\left|d_{i j}\right|^{2} Q_{i j} \\
+\left.2 \pi\left|s_{0}\left(\left|\omega_{j i}\right|^{2}\right) n\left(\left|\omega_{j i}\right|\right)\right| d_{i j}\right|^{2} Q_{i j}
\end{gathered}
$$

Therefore, for $E_{i}>E_{j}$ :

$$
\begin{gathered}
\frac{\Gamma_{i}}{\Gamma_{i}}=\frac{\left|c_{0}\left(\omega_{i j}\right)\right|^{2}\left(n\left(\omega_{i j}\right)+1\right)+\left|s_{0}\left(\omega_{i j}\right)\right|^{2} n\left(\omega_{i j}\right)}{\left|c_{0}\left(\omega_{i j}\right)\right|^{2} n\left(\omega_{i j}\right)+\left|s_{0}\left(\omega_{i j}\right)\right|^{2}\left(n\left(\omega_{i j}\right)+1\right)} \\
=\frac{\left|c_{0}\left(\omega_{i j}\right)\right|^{2}+\left|s_{0}\left(\omega_{i j}\right)\right|^{2} \frac{n\left(\omega_{i j}\right)}{n\left(\omega_{i j}\right)+1}}{\left|c_{0}\left(\omega_{i j}\right)\right|^{2} \frac{n\left(\omega_{i j}\right)}{n\left(\omega_{i j}\right)+1}+\left|s_{0}\left(\omega_{i j}\right)\right|^{2}}
\end{gathered}
$$

Let us consider two typical cases for the choice of $c_{k}$ and $n_{k}$. Of course, it is always possible to consider other cases which are combination of the following two situations. 


\section{1 squeezed vacuum environment}

The simplest case corresponds to the squeezing of the vacuum state. In this case, since $n(k)=0$, we get

$$
\mathcal{N}(k)=\left|c_{k}\right|^{2}-1=\left|s_{k}\right|^{2}
$$

Clearly, it is always possible to choose $c_{k}$ so that $\mathcal{N}(k)$ satisfies (57). This means that we squeeze the $a_{k}$-field with a mode-dependent squeezing parameter.

\section{2 non-squeezed but non-linear temperature state}

We can also think non-squeezed but non-linear temperature state. In this case, since $c_{k}=1\left(s_{k}=0\right)$ we get

$$
\mathcal{N}(k)=n(k) .
$$

and again it is always possible to chose the density $n(k)$ so that $\mathcal{N}(k)$ satisfies (57). Comparing (58) and (59), one can see the similarity of the roles of squeezing and number density modification, which is the replacement of $\left|c_{k}\right|^{2}$ by $n(k)+1$ in (58).

Notice that, when the target state is a thermal equilibrium state for $H_{S}$, i.e.

$$
\frac{\tilde{p}_{j}}{\tilde{p}_{i}}=e^{\beta\left(E_{i}-E_{j}\right)},
$$

from condition (57),

$$
N_{i j}=\frac{1}{\frac{\tilde{p}_{j}}{\tilde{p}_{i}}-1}=\frac{1}{e^{\beta\left(E_{i}-E_{j}\right)}-1}=\frac{1}{e^{\beta \omega\left(k_{i j}\right)}-1},
$$

where $\omega\left(k_{i j}\right)=\omega_{i j}=E_{i}-E_{j}$. This tells us that a thermal state of the (free) environment at a given temperature drives the system to thermal state for its free Hamiltonian at the same temperature. In the general case, $n(k)$ is not the usual Gibbs factor but something else which can be described by a non-linear temperature $\beta(\omega(k))$ as

$$
n(k)=\frac{1}{e^{\beta(\omega(k)) \cdot \omega(k)}-1}
$$

Because of the assimilation Lemma (1) this is equivalent to the following relation for the target state.

$$
\frac{\tilde{p}_{j}}{\tilde{p}_{i}}=e^{\beta\left(\omega\left(k_{i j}\right)\right) \cdot \omega\left(k_{i j}\right)} .
$$

So the nonlinear temperature function describing the stationary state of the system is the same one describing the control state of the environment. In this sense we say that the non-linear temperature environment drives the system to the same non-linear temperature state of the field. Notice that this nontrivial "assimilation" occurs as a consequence of an appropriate choice of the coupling to the environment, i.e. of the $d_{j i}$ whereas in the linear case of a temperature environment (i.e. an usual equilibrium state) this is a quite general phenomenon[3, 11], (See also [14].) If the $d_{j i}$ don't satisfy condition (56), the non-linear temperature environment drives the system to a different stationary state. (See also [11].) 


\section{Purification}

In this section, we discuss the case where the target state is pure,i.e.

$$
\tilde{p}_{0}=1, \quad \tilde{p}_{1}=\tilde{p}_{2}=\cdots \tilde{p}_{N}=0
$$

for $E_{0}<E_{1}<E_{2}<\cdots<E_{N}$.

One can get such a pure state with the control procedure which we discussed in this paper. From conditions (57) and (56), we choose $d_{i 0}$ and $N_{i 0}$ as

$$
\begin{aligned}
\left|d_{i 0}\right|^{2} & =\frac{\Gamma}{Q_{i 0}}, \\
N_{i 0} & =0 .
\end{aligned}
$$

As for the other $d_{i j}$ and $N_{i j}$, we choose as

$$
\left|d_{i j}\right|^{2} N_{i j} Q_{i j}=0 .
$$

Notice that (67) meets with $d_{i j}$ chosen as (56), as far as $N_{i j}$ and $Q_{i j}$ are finite. Threfore the sufficient conditions for this purification is (56). Under these conditions, the state of the system converges to the lowest eigenstate $\left|\mu_{0}\right\rangle\left\langle\mu_{0}\right|$, obeying the master equation (36). This process can be understood as cooling the system by the vacuum. The energy which the initial state of the system has is released to the environment throught the coupling given by (65). Notice that, since $d_{i j}=0$ for $j \neq 0, N_{i j}$ doesn't affect this dynamics for $j \neq 0$. In other words, the initial energy is released by the direct transition from some energy level to the lowest eigenstate.

However, as discussed in the previous section, this cooling effect by the vacuum environment is more universal. As the thermal environment has assimilation property for a a large class of $d_{i j}$, the vacuum environment generally drives the system to the lowest eigenstate of the $H_{S}$. (In fact, the vacuum environment is the low temperature limit of the thermal state.) From this point of view, the specific choice for $d_{i j}$ is not necessary for this cooling procedure. The necessary and sufficient condition for this cooling is the same with the condition for the assimilation in a linear temperature case (see previous section). Therefore $d_{i j} \neq 0$ for all $i \neq j$ is a sufficient condition for this thermal (vacuum) assimilation. Once one can prepare $\mathcal{N}(k)=0$ (it means vacuum state of the environment), the state of the system is driven to the lowest energy eigen state through the proper but much more general interaction which satisfies the condition [14].

\section{Example: Entangled state with Spin-Boson model}

Let us illustrate our technique with one important example, that is, driving to the singlet state a system made of two $1 / 2$-spin particles.

We consider the system Hamiltonian

$$
H_{S}=\sum_{j=1}^{4} \epsilon_{j}\left|\phi_{j}\right\rangle\left\langle\phi_{j}\right|
$$


where

$$
\begin{aligned}
&\left|\phi_{1}\right\rangle=\frac{1}{\sqrt{2}}\left(|+\rangle_{A}|-\rangle_{B}+|-\rangle_{A}|+\rangle_{B}\right) \\
&\left|\phi_{2}\right\rangle=\frac{1}{\sqrt{2}}\left(|+\rangle_{A}|-\rangle_{B}-|-\rangle_{A}|+\rangle_{B}\right) \\
&\left|\phi_{3}\right\rangle=\frac{1}{\sqrt{2}}\left(|+\rangle_{A}|+\rangle_{B}+|-\rangle_{A}|-\rangle_{B}\right) \\
&\left|\phi_{4}\right\rangle=\frac{1}{\sqrt{2}}\left(|+\rangle_{A}|+\rangle_{B}-|-\rangle_{A}|-\rangle_{B}\right) . \\
& \sigma_{z}^{(j)}| \pm\rangle_{j}= \pm| \pm\rangle_{j}, \quad j=A, B .
\end{aligned}
$$

Notice that the Hamiltonian (68) can be rewritten as

$$
H_{S}=\alpha \sigma_{x}^{(A)} \otimes \sigma_{x}^{(B)}+\beta \sigma_{y}^{(A)} \otimes \sigma_{y}^{(B)}+\gamma \sigma_{z}^{(A)} \otimes \sigma_{z}^{(B)}+\delta
$$

i.e. like a standard Heisenberg model, where

$$
\begin{aligned}
& \sigma_{x}^{(j)}|R\rangle_{j}=|R\rangle_{j}, \quad \sigma_{x}^{(j)}|L\rangle_{j}=-|L\rangle_{j}, \quad \sigma_{y}^{(j)}|U\rangle_{j}=|U\rangle_{j}, \quad \sigma_{y}^{(j)}|D\rangle_{j}=-|D\rangle_{j} \\
& |R\rangle_{j}=\frac{1}{\sqrt{2}}\left(|+\rangle_{j}+|-\rangle_{j}\right), \quad|L\rangle_{j}=\frac{1}{\sqrt{2}}\left(|+\rangle_{j}-|-\rangle_{j}\right), \\
& |U\rangle_{j}=\frac{1}{\sqrt{2}}\left(|+\rangle_{j}-i|-\rangle_{j}\right), \quad|D\rangle_{j}=\frac{1}{\sqrt{2}}\left(|+\rangle_{j}+i|-\rangle_{j}\right), \\
& \alpha=\frac{+\epsilon_{1}-\epsilon_{2}+\epsilon_{3}-\epsilon_{4}}{4}, \quad \beta=\frac{+\epsilon_{1}-\epsilon_{2}-\epsilon_{3}+\epsilon_{4}}{4} \\
& \gamma=\frac{-\epsilon_{1}-\epsilon_{2}+\epsilon_{3}+\epsilon_{4}}{4}, \quad \delta=\frac{+\epsilon_{1}+\epsilon_{2}+\epsilon_{3}+\epsilon_{4}}{4}
\end{aligned}
$$

or

$$
\begin{array}{ll}
\epsilon_{1}=+\alpha+\beta-\gamma+\delta, & \epsilon_{2}=-\alpha-\beta-\gamma+\delta \\
\epsilon_{3}=+\alpha-\beta-\gamma+\delta, & \epsilon_{4}=-\alpha+\beta+\gamma+\delta .
\end{array}
$$

Once one can control the parameters $\alpha, \beta, \gamma$, (and $\delta$ ), one can always prepare the Hamiltonian so that it becomes generic in the sense of (21).

\begin{tabular}{|c||c|c|c|c|}
\hline$\omega_{i j}=\epsilon_{i}-\epsilon_{j}$ & $i=1$ & $i=2$ & $i=3$ & $i=4$ \\
\hline \hline$j=1$ & 0 & $-2(\alpha+\beta)$ & $-2 \beta$ & $-2(\alpha-\gamma)$ \\
\hline$j=2$ & $+2(\alpha+\beta)$ & 0 & $+2 \alpha$ & $+2(\beta+\gamma)$ \\
\hline$j=3$ & $+2 \beta$ & $-2 \alpha$ & 0 & $-2(\alpha-\beta-\gamma)$ \\
\hline$j=4$ & $+2(\alpha-\gamma)$ & $-2(\beta+\gamma)$ & $+2(\alpha-\beta-\gamma)$ & 0 \\
\hline
\end{tabular}

We write the target state as $\mu=\sum_{j} p_{j}\left|\phi_{j}\right\rangle\left\langle\phi_{j}\right|$ with

$$
p_{1} \geq p_{2} \geq p_{3} \geq p_{4} .
$$

The conditions for $H_{S}$ we listed in the end of sec. 4 require to choose the parameter $\alpha, \beta, \gamma, \delta$ so that

$$
\epsilon_{1}<\epsilon_{2}<\epsilon_{3}<\epsilon_{4} \text {. }
$$


As for the interaction Hamiltonian with a bosonic environment, we take

$$
\begin{aligned}
H_{I} & =D \int d k g(k) a_{k}^{\dagger}+h . c . \\
& =\sum_{i \neq j} \int d k g_{i j}(k)\left|\phi_{i}\right\rangle\left\langle\phi_{j}\right| a_{k}^{\dagger}+h . c .
\end{aligned}
$$

where

$$
D=\sum_{i \neq j} d_{i j}\left|\phi_{i}\right\rangle\left\langle\phi_{j}\right|, \quad g_{i j}(k)=\left\langle\phi_{i}|D| \phi_{j}\right\rangle g(k)=d_{i j} g(k) .
$$

The free Hamiltonian of the boson-field is

$$
H_{B}=\int d k \omega(k) a_{k}^{\dagger} a_{k}, \quad\left[a_{k}, a_{k^{\prime}}^{\dagger}\right]=\delta\left(k-k^{\prime}\right) .
$$

From (57), the proper $d_{i j}$ for the control is obtained as

$$
d_{i j}=e^{i \theta_{i j}}\left(\frac{\Gamma}{Q_{i j}}\right)^{1 / 2} \sqrt{p_{i}-p_{j}}, \text { for } i>j .
$$

When $p_{i} \neq p_{j}$, the initial state of the field should satisfy

$$
N_{i j}=\frac{p_{i}}{p_{j}-p_{i}} .
$$

with $N_{i j}$ given by $(47),(44),(40)$ and (18). Now let us consider the case when the target state is $\left|\phi_{1}\right\rangle\left\langle\phi_{1}\right|$. From the discussion in the previous section, we find that

$$
d_{1 j}=e^{i \theta_{1 j}}\left(\frac{\Gamma}{Q_{1 j}}\right)^{1 / 2}, \quad j=2,3,4, \quad \text { and } \quad \text { other } d_{i<j}=0
$$

is a sufficient condition. In addition, for simplicity, we assume that

$$
Q_{1 j}=Q(\text { i.e. it does not depend on } j \text { ). }
$$

In this case one possible choice of $D$ is for example

$$
D=\sqrt{\frac{\Gamma}{Q}} \sum_{j=2,3,4}\left|\phi_{1}\right\rangle\left\langle\phi_{j}\right| .
$$

Notice the following condition holds:

$$
\Gamma=\frac{1}{3} Q\left\langle\phi_{1}\left|D D^{\dagger}\right| \phi_{1}\right\rangle .
$$

This tells us that the life time of the initial state $\Gamma^{-1}$ depends on the choice of operator $D$. More precisely, the speed of the convergence is proportional to the norm of $D^{\dagger}\left|\phi_{1}\right\rangle$. In the general case without the additional condition (86), the $\Gamma$ is constrained as

$$
\frac{1}{3} Q^{(m)}\left\langle\phi_{1}\left|D D^{\dagger}\right| \phi_{1}\right\rangle \leq \Gamma \leq \frac{1}{3} Q^{(M)}\left\langle\phi_{1}\left|D D^{\dagger}\right| \phi_{1}\right\rangle
$$

where

$$
Q^{(m)}=\operatorname{Min}_{j} Q_{1 j}, \quad Q^{(M)}=\operatorname{Max}_{j} Q_{1 j} .
$$




\section{Acknowledgment}

One of the authors (KI) is grateful to the Centro Vito Volterra for kind hospitality. This work is partially supported by JSPS for KI.

\section{Appendix}

Introducing a pair of independent creation and annihilation operators $\xi_{1}(k)$, $\xi_{1}^{\dagger}(k), \xi_{2}(k), \xi_{2}^{\dagger}(k)$ with commutaion relations

$$
\left[\xi_{i}(k), \xi_{j}^{\dagger}\left(k^{\prime}\right)\right]=\delta_{i j} \delta\left(k-k^{\prime}\right)
$$

acting in a Fock space $\mathcal{H}_{0}$ with vacuum vector $\Phi_{0}$

$$
\xi_{i}(k) \Phi_{0}=0, \quad i=1,2,
$$

the GNS representation of the $a_{k}, a_{k}^{\dagger}$ - field will respect to the state (18) becomes

$$
\begin{aligned}
& \tilde{a}_{k}=\sqrt{n(k)+1} \xi_{1}(k)+\sqrt{n(k)} \xi_{2}^{\dagger}(k) \\
& \tilde{a}_{k}^{\dagger}=\sqrt{n(k)+1} \xi_{1}^{\dagger}(k)+\sqrt{n(k)} \xi_{2}(k) .
\end{aligned}
$$

The Schrödinger equation in the interaction picture is therefore

$$
\frac{d}{d t} U_{t}^{(\lambda)}=-i \lambda H_{I}(t) U_{t}^{(\lambda)}
$$

where

$$
\begin{aligned}
& H_{I}(t)= e^{i H_{0} t} H_{I} e^{-i H_{0} t} \\
&= \sum_{i j} \int d k g_{i j}^{*}(k)\left|\mu_{j}\right\rangle\left\langle\mu_{i}\right| a_{k} e^{-i\left(\omega(k)-\omega_{j i}\right) t}+h . c . \\
&= \sum_{i j} \int d k g_{i j}^{*}(k) L_{i j}^{\dagger}\left\{c_{k}^{*}\left(\sqrt{n(k)+1} \xi_{1}(k)+\sqrt{n(k)} \xi_{2}^{\dagger}(k)\right)\right. \\
&\left.\quad+s_{k}\left(\sqrt{n(-k)+1} \xi_{1}^{\dagger}(-k)+\sqrt{n(-k)} \xi_{2}(-k)\right)\right\} e^{-i\left(\omega(k)-\omega_{j i}\right) t} \\
& \quad+h . c . \\
&= \sum_{i j} L_{i j}^{\dagger}\left(\Xi_{c, i j}^{(-)}(t)+\Xi_{s, i j}^{(-)^{\dagger}}(t)+\Xi_{c, i j}^{(+)^{\dagger}}(t)+\Xi_{s, i j}^{(+)}(t)\right)+h . c . \\
& \Xi_{c, i j}^{(-)}(t)=\int d k g_{i j}^{*}(k) c_{k}^{*} \sqrt{n(k)+1} \xi_{1}(k) e^{-i\left(\omega(k)-\omega_{j i}\right) t} \\
& \Xi_{s, i j}^{(-)^{\dagger}}(t)=\int d k g_{i j}^{*}(k) s_{k} \sqrt{n(-k)+1} \xi_{1}^{\dagger}(-k) e^{-i\left(\omega(k)-\omega_{j i}\right) t} \\
& \Xi_{c, i j}^{(+)}(t)=\int d k g_{i j}^{*}(k) c_{k}^{*} \sqrt{n(k)} \xi_{2}^{\dagger}(k) e^{-i\left(\omega(k)-\omega_{j i}\right) t} \\
& \Xi_{s, i j}^{(+)}(t)=\int d k g_{i j}^{*}(k) s_{k} \sqrt{n(-k)} \xi_{2}(-k) e^{-i\left(\omega(k)-\omega_{j i}\right) t}
\end{aligned}
$$


The stochastic limit describes the quantum dynamics in the regime of weak coupling $(\lambda \rightarrow 0)$ and large times $\left(t \rightarrow t / \lambda^{2}\right)$. Under rescaling the time $t \mapsto t / \lambda^{2}$, the Schrödinger equation becomes

$$
\frac{d}{d t} U^{(\lambda)}\left(t / \lambda^{2}\right)=-i \frac{1}{\lambda} H_{I}\left(t / \lambda^{2}\right) U^{(\lambda)}\left(t / \lambda^{2}\right) .
$$

and the quantum field undergoes a frequency dependent rescaling

$$
a_{k} \rightarrow \frac{1}{\lambda} e^{-i \frac{t}{\lambda^{2}}(\omega(k)-\omega)} a_{k}
$$

The main result of this theory is expressed by the stochastic golden rule $[3,4,5]$ according to which in the limit $\lambda \rightarrow 0$ :

1. for each Bohr frequency $\omega$, the rescaled field (101) becomes a quantum white noise (or master field) $b_{\omega}(t, k)$ satisfying the commutation relations

$$
\left[b_{\omega}(t, k), b_{\omega^{\prime}}^{\dagger}\left(t^{\prime}, k^{\prime}\right)\right]=\delta_{\omega \omega^{\prime}} 2 \pi \delta\left(t-t^{\prime}\right) \delta(\omega(k)-\omega) \delta\left(k-k^{\prime}\right)
$$

2. the solution $U^{(\lambda)}\left(t / \lambda^{2}\right)$ converges to the solution of the white noise equation

$$
\frac{d}{d t} U(t)=-i h(t) U(t)
$$

where the white noise Hamiltonian is given by

$$
h(t)=\sum_{i j} L_{i j}^{\dagger}\left(b_{c, i j}^{(-)}(t)+b_{s, i j}^{(-)^{\dagger}}(t)+b_{c, i j}^{(+)^{\dagger}}(t)+b_{s, i j}^{(+)}(t)\right)+h . c .
$$

and the master fields (white noises) are

$$
b_{x, i j}^{(\varepsilon)}(t)=\lim _{\lambda \rightarrow 0} \frac{1}{\lambda} \Xi_{x, i j}^{(\varepsilon)}\left(t / \lambda^{2}\right), \quad x=c, s, \quad \varepsilon=\mp .
$$

In this case the commutation relations (102) become:

$$
\left[b_{x, i j}^{(\varepsilon)}(t), b_{y, i^{\prime} j^{\prime}}^{(\varepsilon) \dagger}\left(t^{\prime}\right)\right]=\delta_{x y} \delta_{\varepsilon \varepsilon^{\prime}} \delta_{\omega_{j i} \omega_{j^{\prime} i^{\prime}}} \Gamma_{x, i j}^{(\varepsilon)} \delta\left(t-t^{\prime}\right)
$$

where

In addition, the commutation relation between $U_{t}$ and these noise operators are obtained as

$$
\begin{aligned}
{\left[b_{c, i j}^{(-)}(t), U_{t}\right] } & =\sum_{j^{\prime} i^{\prime}} \delta_{\omega_{j i} \omega_{j^{\prime} i^{\prime}}}\left(\Gamma_{c, i j}^{(-)}-i \Delta_{c, i j}^{(-)}\right) L_{i^{\prime} j^{\prime}} U_{t}, \\
{\left[b_{s, i j}^{(-)}(t), U_{t}\right] } & =\sum_{j^{\prime} i^{\prime}} \delta_{\omega_{j i} \omega_{j^{\prime} i^{\prime}}}\left(\Gamma_{s, i j}^{(-)}+i \Delta_{s, i j}^{(-)}\right) L_{i^{\prime} j^{\prime}}^{\dagger} U_{t}, \\
{\left[b_{c, i j}^{(+)}(t), U_{t}\right] } & =\sum_{j^{\prime} i^{\prime}} \delta_{\omega_{j i} \omega_{j^{\prime} i^{\prime}}}\left(\Gamma_{c, i j}^{(+)}+i \Delta_{c, i j}^{(+)}\right) L_{i^{\prime} j^{\prime}}^{\dagger} U_{t}, \\
{\left[b_{s, i j}^{(+)}(t), U_{t}\right] } & =\sum_{j^{\prime} i^{\prime}} \delta_{\omega_{j i} \omega_{j^{\prime} i^{\prime}}}\left(\Gamma_{s, i j}^{(+)}-i \Delta_{s, i j}^{(+)}\right) L_{i^{\prime} j^{\prime}} U_{t},
\end{aligned}
$$

where

3. From the step 1, by means of a standard procedure one can deduce the Langevin and the master equation (cf. [3]). 


\section{References}

[1] C.H.Bennet et al, Phys. Rev. Lett. 69, 2881 (1992); 70, 1895 (1993).

[2] For example, The Physics of Quantum Information, edited by D. Bouwmeester, A. Ekert, and A. Zeilinger (Springer-Verlag, Heidelberg, 2000), and references therein.

[3] L. Accardi, Y. G. Lu, and I. V. Volovich, Quantum Theory and Its Stochastic Limit Springer-Verlag (2002)

[4] L. Accardi, A. Frigerio, and Y. G. Lu, Commun. Math. Phys. 131, 537 (1990); L. Accardi, J. Gough, and Y. G. Lu, Rep. Math. Phys. 36, 155 (1995); L.Accardi, S.V.Kozyrev, I.V.Volovich, Phys. Rev. A 57 (1997); Phys. Lett. A 260, 31 (1999). G. Kimura, K. Yuasa, and K. Imafuku, Phys. Rev. A 63 (2001), 022103; Phys. Rev. Lett. 89 (2002), 140403.

[5] L. Accardi, F. Fagnola (eds.), Quantum interacting particle systems, Lecture Note of Levico school, September 2000, Volterra Preprint N.431. in Quantum interaction particles L. Accardi, F. Fagnola (eds.) World Scientific (2002).

[6] L. Accardi, A. Boukas, Control of quantum Langevin equations, Open Systems and Information Dynamics (2003); Control of elementary quantum flows, Proceedings of the 2-d Meijo Winter School, Quantum Information and Complexity, T. Hida (ed.), World Scientific (2003)

[7] J.I. Cirac and P. Zoller, Phys. B 64, 623 (1997)

[8] C. Monroe, D.M. Meekhof, B.E. King, W.M. Itano, and D.J. Wineland, Phys. Rev. Lett. 75, 4714 (1995).

[9] P. Domokos, J.M. Raimond, M. Brune, and S. Haroche, Phys. Rev. A 52, 3554 (1995).

[10] A. Beige, D. Braun, B. Tregenna, and P.L. Knight, Phys. Rev. Lett. 85, $1762(2000)$.

[11] L. Accardi, K. Imafuku, "Dynamical detailed balance and local KMS condition for non-equilibrium states" quant-ph/0209088.

[12] Gorini V., Kossakowski A., Sudarshan E.C.G., J. Math. Phys 17, 821-825 (1976).

[13] Lindblad G. Comm. Math. Phys. 48,119-130 (1976).

[14] The necessary and sufficient condition of the interaction for the assimilation by the linear temperature environment (i.e. usual thermalization by equilibrium heat bath) is the generator in R.H.S of (22) generates full algebra on the system space [5]. 